\title{
Nicht-enzymatische Glykosylierung von Kollagenen
}

\author{
R. W. Fischer \\ Eidgenössische Technische Hochschule Zürich
}

\begin{abstract}
Zusammenfassung:
Das aus menschlichen Nieren isolierte Basalmembrankollagen (Kollagen Typ IV) wurde auf den Gehalt an nichtenzymatisch glykosylierten Aminosäuren untersucht. Im Vergleich zu Kollagen aus gesunden Nieren, wurde bei diabetischen Nieren eine um den Faktor 1,6 erhöhte nicht-enzymatische Glykosylierung festgestellt. Die Stöchiometrie ergab Werte um 0,6 Moleküle Glucose pro Kollagenmolekül beim Normalen, bis 1,0 beim Diabetiker.
\end{abstract}

Ein Unterschied in der enzymatischen Glykosylierung konnte nicht beobachtet werden.

Ob nun die nicht-enzymatische Glykosylierung eine strategisch wichtige Position betrifft die die pathophysiologische Verdickung der Basalmembrane erklären kann, muß erst noch gezeigt werden.

\section{Schlüsselwörter:}

Nicht-enzymatische Glykosylierung - Basalmembran - Kollagen - Diabetes mellitus

\begin{abstract}
Summary:
Basementmembrane collagen (collagen typ IV) was isolated from normal human kidneys and the proportion of nonenzymatically glycosylated amino acids was determined. Collagen from diabetic human kidneys was similarly isolated and the nonenzymatic glycosylation was increased by a factor of 1.6. A stoichiometric calculation resulted in ratio of 0.6 mole glucose per collagen molecule for the normals up to 1.0 for the diabetics.
\end{abstract}

There was no difference in enzymatic glycosylation detected. Whether this nonenzymatic glycosylation affects a strategic important position which could explain the basement membrane thickening has still to be shown.

\section{Keywords:}

Nonepzymatic glycosylation - basement membrane - collagen - diabetes mellitus

Die nicht-enzymatische Glykosylierung findet zwischen den Aminogruppen der Proteine einerseits, und der Karbonylfunktion der Aldolasen und Ketosen andererseits, statt. Dieser absichtlich weitgefaßte Begriff soll zeigen, daß das Phänomen der nicht-enzymatischen Glykosylierung ubiquitär ist. Einige Voraussetzungen müssen im Hinblick auf die Ausbeute dieser chemischen Kondensationsreaktion jedoch gegeben sein. Es sind dies:

Für den Zucker

- hohe Konzentration

- Karbonylform

Für das Protein

- lange biologische Halbwertszeit

- freie Aminogruppen

Für die Aminogruppen

- tiefer pK-Wert

- Zugang zur hohen Zuckerkonzentration

- keine sterische Hinderung.

Der pK-Wert der Aminogruppe ist stark von seiner unmittelbaren Umgebung abhängig. Diese Tatsache läßt sich am Beispiel von Valin, der $\mathrm{N}$-terminalen Aminosäure der $\beta$-Kette im menschlichen Hämoglobin, zeigen. Der pK-Wert der $\mathrm{NH}_{2}-$ Gruppe dieser Aminosäure in reinem, isolierten Zustand beträgt 9,69. Im Hämoglobin, wo diese Aminogruppe auch nicht-enzymatisch glykosyliert wird $\left(\mathrm{HbA}_{1 \mathrm{c}}\right)$, wurden pKWerte von 7,05 in CO-ligandiertem Zustand, bzw. 6,84 in
Deoxyhämoglobin, gemessen. Daß allein schon die allosterische Konformationsänderung, bedingt durch den Ligandenwechsel, eine Änderung des pK's verursacht, unterstreicht die Bedeutung der Tertiärstruktur eines Proteins. Aus diesem Grunde darf dem pK-Wert der $\varepsilon$-Aminogruppe des Lysins in seiner isolierten Form von 10,53 keine allzu große Bedeutung beigemessen werden.

In der Tabelle 1 sind drei Hexosen mit einigen für die nichtenzymatische Glykosylierung relevanten Eigenschaften aufgeführt.

Es ist ersichtlich, daß die Zucker nur in ihrer offenkettigen Form mit den Aminogruppen reagieren, denn die Reaktionskinetik ist um so schneller, je größßer der in der Karbonylform vorliegende Anteil des betreffenden Zuckers ist. Korrigiert man für den unterschiedlichen Anteil der offenen Form in den verschiedenen Zuckern, so läßt sich erkennen, daß die Ketosen etwa $50 \mathrm{mal}$ langsamer als die vergleichbaren Aldosen mit den Aminogruppen reagieren. In den physiologischen Systemen schließlich ist die Konzentration der Kohlenhydrate der entscheidende Faktor. Demzufolge ist die Glucose der Kandidat, der sich für die nicht-enzymatische Glykosylierung anbietet.

Neben anderen Veränderungen zeigt der Diabetiker eine deutlich, bis zu 10mal, verdickte Basalmembran (2). Eine Basalmembran besteht zwischen 40 und $80 \%$ aus Kollagen. Dieses Protein hat sowohl eine lange Halbwertszeit von etwa 
Tab. 1:

\begin{tabular}{|c|c|c|c|c|}
\hline \multirow{2}{*}{$\begin{array}{r}\text { Zucker } \\
\text { Typ }\end{array}$} & \multirow[t]{2}{*}{ \% Karbonylform } & \multirow{2}{*}{$\begin{array}{l}\text { Reaktivität mit Hämoglobin } \\
\text { (aus Lit 1) } \\
k, \times 10^{-3}\left(\mathrm{mM}^{-1} \mathrm{hr}^{-1}\right)\end{array}$} & \multicolumn{2}{|c|}{ Physiologische Konzentration } \\
\hline & & & Normal & Diabetisch \\
\hline $\begin{array}{l}\text { Glucose } \\
\text { Aldose }\end{array}$ & 0,002 & 0,6 (1) & $5 \mathrm{mM}$ & $5-20 \mathrm{mM}$ \\
\hline $\begin{array}{l}\text { Galaktose } \\
\text { Aldose }\end{array}$ & 0.02 (10fach) & $2,8(4,6$ fach $)$ & $0.04 \mathrm{mM}$ & - \\
\hline $\begin{array}{l}\text { Fiuktose } \\
\text { Ketose }\end{array}$ & 0.7 (350fach) & 4,5 (7,5fach) & $0.04 \mathrm{mM}$ & - \\
\hline
\end{tabular}

2,5 Jahren, als auch viele Lysin- bzw. Hydroxylysinreste, die nicht-enzymatisch glykosyliert werden können.

Kollagen ist ein stäbchenförmiges Molekül das aus drei einzelnen Ketten zusammengesetzt ist. Jede dieser Ketten beschreibt eine linksdrehende Helix. Damit eine Helix mit drei Aminosäuren pro Windung gebildet werden kann, nimmt aus sterischen Gründen Glycin mit einem Wasserstoffatom als Seitenkette jede dritte Position ein. Weitere 25\% der Aminosäuren werden von Prolin beansprucht. Prolin ist für die starre Konformation verantwortlich, in seiner hydroxylierten Form bildet es außerdem stabilisierende Wasserstoffbrücken aus. Die aromatischen Aminosäuren wie Tryptophan und Tyrosin fehlen im Kollagen vollständig.

Bei der Biosynthese werden die einzelnen Ketten von den Ribosomen direkt in das Lumen des rauhen endoplasmatischen Retikulums sezerniert. Bevor die Synthese abgeschlossen ist, werden die entstehenden Ketten enzymatisch glykosyliert und hydroxyliert. Besitzen die einzelnen Ketten etwa 90 Hydroxyprolinreste, so kombinieren sie zum tripelhelikalen Molekül. Die Hydroxylasen und die Galactosyltransferase können an der Trypelhelix nicht mehr angreifen, somit sind diese enzymatischen Modifikationen beendet. $\mathrm{Zu}$ diesem Zeitpunkt besitzt das Kollagen an seinen Enden die nichthelikalen Prokollagenverlängerungen, welche dem Molekül eine gute Löslichkeit verleihen. Via den Golgikomplex wird das Prokollagen in den Extrazellulärraum ausgeschieden. Peptidasen spalten hier die Prokollagenenden ab und hinterlassen das native Molekül, welches nur noch kurze nichthelikale Sequenzen an seinen Enden aufweist. Die Lysyloxidase sorgt nun dafür, daß einige Lysin- bzw. Hydroxylysinreste oxidativ deamidiert werden (3). Die so gebildeten Aldehydfunktionen können untereinander kovalente Quervernetzungen ausbilden, und die Iosen Fibrillen stabilisieren.

Die Fibrillenbildung und die Quervernetzung wurden auch zuerst im Zusammenhang mit der nicht-enzymatischen Glykosylierung untersucht (4). Die Autoren fanden mit zunehmender Glykosylierung eine verzögerte Fibrillenbildung sowie das Fehlen der stabilisierenden Quervernetzung.

Das Basalmembrankollagen Typ IV weist gegenüber den interstitiellen Kollagenen, Typ I, II und III, einige wesentliche Unterschiede auf. So ist seine Trypelhelix unterbrochen, das Molekül kann, neben Kollagenase, auch von anderen Proteasen gespalten werden. Im nativen Kollagen Typ IV sind ferner die Prokollagenenden nur unvollständig oder gar nicht abgespalten. Diese nichthelikalen Anteile sind auch für die Bildung des Netzwerkes verantwortlich (5). Die Verknüpfungsregion von vier Kollagenmolekülen wird als 7S-Domäne bezeichnet und ist stark über Disulfidbrücken vernetzt. Man muß sich also stets bewußt sein, daß Untersuchungen an diesem Basalmembrankollagen nur an Fragmenten vorgenommen werden können.

Untersuchungen von menschlichem Kollagen Typ IV, das aus normalen und diabetischen Nieren isoliert wurde (6), zeigt als erstes Resultat, daß bezogen auf das Feuchtgewicht bei den diabetischen Nieren mehr Kollagen isoliert wird.

Bei den enzymatischen Modifikationen wie Hydroxyprolin, Hydroxylysin und $\mathrm{O}$-glykosidisch gebundenen Hexosen konnte kein Unterschied festgestellt werden.

Die nicht-enzymatische Glykosylierung wurde mit verschiedenen Methoden bestimmt und die Stöchiometrie berechnet. Der Einbau von Tritium nach Reduktion mit markiertem Natriumborhydrid zeigte eine 1,6fache Erhöhung bei dem diabetischen Kollagen. Die Auftrennung auf dem Aminosäurenanalysator nach saurer Hydrolyse ließ für die modifizierten Aminosäuren Lysin und Hydroxylysin dasselbe Verhältnis erkennen. Von der aufgetragenen Radioaktivität wurden 45\% unter den Peaks dieser Aminosäuren gefunden. Interessant ist auch, daß die $55 \%$ Restaktivität, die auf dem Analysator nicht zurückgehalten wurden (Elution im äußeren Säulenvolumen) beim Diabetiker ebenfalls eine 1,6fache Erhöhung zeigten.

Die Auftrennung des Hydrolysates auf einer Boronataffinitätsmatrix, bei der die Moleküle mit benachbarten $\mathrm{OH}$-Gruppen (Zucker) zuerst gebunden werden und anschließend mit Sorbitol eluiert werden, ergab ebenfalls eine 1,6fache Erhöhung beim Diabetiker.

Die TBA-Farbreaktion schließlich stellte eine 1,7fache Erhöhung fest.

Berechnungen des molaren Verhältnisses ergab Werte von $0,6 \mathrm{Mol}$ Glucose pro Mol Kollagen beim normalen bzw. 1,0 beim diabetischen Kollagen.

Zusammenfassend kann festgestellt werden: Eine nicht-enzymatische Glykosylierung von Kollagen findet statt; sie ist beim Diabetiker etwa doppelt so hoch, ob es strategisch wichtige Positionen betrifft, muß erst noch gezeigt werden.

Für die pathophysiologischen Veränderungen der Basalmembran konnte bis heute der direkte Zusammenhang mit der nicht-enzymatischen Glykosylierung noch nicht nachgewiesen werden.

\section{Schriftum:}

1. BUNN, H. F., HIGGINS, P. J.: Reaction of monosaccharides with proteins: Possible Evolutionary Significance. Science 213, 222-224 (1981).

2. VRACKO R.: Front. Matrix Biology. S. Karger, Basel, 7, 78-89 (1979) 3. GAY, S., MILLER, E.: Collagen in the physiology and pathology of connective tissue. Gustav Fischer, Stuttgart

4. GUITTON, J. D., LE PAPE, A., SIZARET, P. Y., MUH, J. P.: Effects of in vitro. $\mathrm{N}$-glucosylation on type-I collagen fibrillogenesis. Bioscience Reports 1, 945-954 (1981). 5. SCHUPPAN, D. TIMPL, R., GLANVILLE, R.: Discontinuities in the triple helical sequence GLY-X-Y of basement membrane (Type-IV) collagen. FEBS Lett. 115, 297-300 (1980) 6. TRÜEB, B., FLÜCKIGER, R., WINTERHALTER, K. H.: Nonenzymatic glycosylation of basement membrane collagen in Diabetes mellitus. Submitted.

Anschrift des Verfassers:

René W. Fischer

Eidgenössische Technische Hochschule

Laboratorium für Biochemie I

ETH-Zentrum

$\mathrm{CH}-8092$ Zürich 


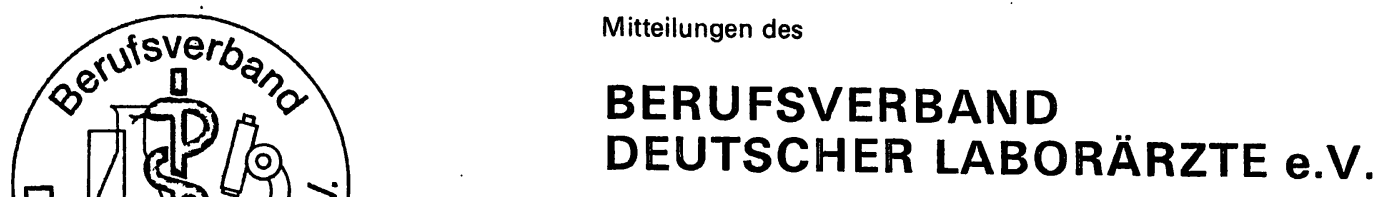

Heft 2/1984, Seite $13-20$

\title{
Die Kosten-Nutzen-Relation von Laboruntersuchungen*
}

\author{
Otto Fenner
}

Über die Kosten-Nutzen-Relation von Laboruntersuchungen in der Medizin zu reden, ist eine undankbare Aufgabe. Bisher gibt es dafür keine sichere Grundlage. Zu verschieden sind die Blickwinkel, aus denen man beides, Kosten sowohl wie Nutzen betrachten könnte. Laboratorien sind Brutstätten neuer Entwicklungen. Diese Aussage gilt im Guten-Nützlichen wie im Bösen-Schädlichen. Auf Laboratorien ist nicht verzichtbar, in den Laboratorien muß mit einem hohen Bewußtsein der Mißbrauchsmöglichkeiten gearbeitet werden. Erkenntnisse im Laboratorium sind wertfrei. Bedeutung erlangen sie erst durch unsere menschliche Ausdeutung. Diese kann falsch oder richtig sein (Beispiel: Atomenergie Atombombe). Meistens sind Erkenntnisse keine Einzelleistung oder Beobachtung, sie entwickeln sich über einen längeren Zeitraum mit Abwegen, Fehlschlägen und unendlichen Mühen, ehe sie reifen.

Ansichten, die heute noch Lehrmeinung waren und für absolut sicher galten, werden schon bald verworfen. Man kann damit rechnen, daß alle 3-5 Jahre ein wesentlicher Umsatz in der Beurteilung von Methoden und Geräten erfolgt. Entsprechend sind die Kosten hoch, eine Geräteentwicklung wird noch oft vor ihrer Amortisation abgebrochen.

Dennoch bleiben gewisse Standards, die zur allgemeinen Anwendung kommen.

Als Beispiel die Blutzuckeruntersuchung: Wer nüchtern mehr als $130 \mathrm{mg} / \mathrm{dl}$ Zucker im Blut hat, ist verdächtig, einen Diabetes zu haben. Die Blutzuckerbestimmung hat seit 50 Jahren unverändert ihre Aussagekraft behalten, gewandelt haben sich seit Hagedorn und Crecelius nur die Methoden des Nachweises. Sie sind heute dank der Hilfe der Laboratorien der Industrie jedem zugänglich. Ich sage bewußt: jedem. Der Patient kann heute seinen Blutzucker in einem Tropfen Blut einfach und schmerzlos selbst messen. - Wie groß ist der Nutzen? Für den einzelnen Patienten unabschätzbar groß. Er lebt so lange, wie Gott inm das erlaubt, oder modern gesagt, so lange, wie seine genetische Disposition vorgeplant ist. Mit Hilfe einer ganz einfachen Laboruntersuchung kann er seinen

\footnotetext{
- Vortrag gehalten am 30.11.1983 auf einer Pressekonferenz der Deutschen Gesellschaft für Laboratoriumsmedizin während der MEDICA 83 in Düsseldorf mit dem Thema: Das ärzlliche Laboratorium - Bedeutung für die medizinische Diagnostik
}

Mangel beheben und den Insulinbedarf so regeln, daß keine Schäden auftreten.

Entsprechend ist die Blutzuckeruntersuchung Spitzenreiter in der Häufigkeitsskala. Die Kosten-Nutzen-Relation ist ausgeglichen. - Die Untersuchung ist erprobt, die Aussage sicher, die Anwendung einfach, der Nutzen groß und die Einzelkosten sind gering. - Die Unterlassung der Untersuchung wäre nicht nur schädlich, sondern in ihrer Folge tödlich. Die Untersuchung ist also indiziert.

Dennoch ist die Durchführung zur Zeit in Frage gestellt. Die Gesamtkosten-Entwicklung im Laboratorium ist infolge Mengenausweitung ungebremst. Bei gestoppten Honoraren und einem begrenzten Finanzvolumen muß jede Mehrleistung den Geldwert der Einzelleistung senken. Unterhalb einer bestimmten Schwelle sind die Kosten nicht mehr gedeckt, die Leistung ist nicht erbringbar; das ist trotz aller Rationalisierungsmaßnahmen zur Zeit zu befürchten. - Auch das Rezept der Konzentrierung zieht hier nicht. Der Blutzucker muß in der ärtlichen Praxis erbringbar sein. - Die Kriterien des Wettbewerbs sind nicht nur hier, sondern bei allen ärtlichen Handlungen unsinnig.

Maßstab kann nur die Notwendigkeit, die Indikation sein! Das Wort Wettbewerb ist gefallen. Der Staat hat die Frage gestellt, die Wanderschäfer haben den Tip aufgegriffen. Als Wanderschäfer werden solche Laboratorien charakterisiert, die Reklame machen und überall Proben einsammeln und sich für die ärztliche Berufsausübung unter Mißachtung der gesetzlichen Regelungen atypisch verhalten. - Die Zahl der Laborleistungen wird durch Beteiligung am Honorar außerhalb der Indikation gesteigert. Der Zwischengewinn wird dem Veranlasser als Anreiz zur vermehrten Abnahme angeboten. - Nicht der Verbraucher (Patient), sondern der Mittler (Arzt) ist also der Interessierte. Nun beginnt sich die Kosten-Nutzen-Skala zu verschieben, obwohl der Nutzen der Untersuchung an sich außer Zweifel bleibt. Eine unnützliche Mengenausweitung wird durch „Wettbewerb" unter Korrumpierung des Mittlers künstlich erzeugt. Die Kosten-Nutzen-Bilanz wird defizitär. Dies ist beispielhaft für die Entwicklung in der gesamten medizinischen Chemie im Bereich der ambulanten Versorgung. Der Schaden der Kosten-Nutzungs-Verschiebung für die Sozialversicherung ist dabei so beträchtlich, daß nach Regulativen gesucht werden muß. 
Ich schätze, daß durch die Rückkehr zur Indikation etwa 200 Millionen DM jährlich gespart werden könnten. Damit konnte der Punktwert um 10\% steigen, d.h. von 7 auf 8 oder von 9 aul 10 Pfennig. Das hätte den Vorteil, daß auch Laborleistungen, die heute schon nicht mehr erbringbar sind, wieder kostendeckend bezahll würden.

Ausschließlich die Indikation und das Sicherheitsbedürfnis von Patient und Arzt rechtfertigen die in der Krankenbehandlung zu ergreifenden Maßnahmen. Mit dieser einfachen Aussage könnte das Kapitel Kosten-Nutzen abgeschlossen werden.

Lassen Sie aber noch ein weiteres Beispiel bringen:

1957 schlossen die Ersatzkassen und die KBV in Bielefeld cinen Vertrag zur Überwachung der Schwangeren zur Erkennung der Blutgruppenunverträglichkeit. Aus diesem Vertrag, der auf meine Anregung zustande kam, entwickelten sich nach und nach die Muttervorsorgeuntersuchungen, die Anti-D Prophylaxe, die Überwachungen der Rötelninfektion, anderer Viruserkrankungen, der Lues und, soweit nötig, der Toxoplasmose. Kürzlich hat Prof. Werner im Bundesgesundheitsblatt angeregt, die Toxoplasmoseuntersuchung auf alle Schwangeren auszudehnen. Dabei machte er folgende Kosten-Nutzen-Rechnung:

Jährlich werden 151 toxoplasmosekranke geschädigte Kinder geboren. $75 \%$ dieser Kinder wären durch rechtzeitige Erkennung mit Hilfe einer Laboruntersuchung und anschließender Behandlung der Mutter heilbar.

Um die erkrankten Mütter mit ihren Kindern zu entdecken, müßten 620000 Schwangere jährlich untersucht werden. Die Gesamtkosten dafür betragen 43 Millionen DM. Die Pflegekosten für die kranken Kinder würden 23 Millionen DM betragen. Wirtschaftlich betrachtet wäre die Kosten-NutzenRelation mit 18 Millionen DM defizitär.

Trotz dieser Rechnung empfiehlt Prof. Werner zu Recht aus humanitären Gründen zur Vermeidung des menschlichen Leidens grundsätzlich die Durchführung dieser Maßnahme im Rahmen der Muttervorsorge. Ich teile seine Meinung.

Hier ist die Indikation klar herausgestellt. Die Methoden sind erprobt. Erfahrungen aus Österreich bestätigen dies voll und ganz. - Die wirtschaftliche Kritik kann dem nichts Entscheidendes entgegegensetzen. Es sei denn, unser Volk ist so arm, daß es sich eine solche humanitäre Maßnahme einfach nicht erlauben könnte. - Wie steht es damit?

1980 standen den Kassenärzten 2 Milliarden DM für Laborleistungen zur Verfügung. Zur gleichen Zeit wurden 4 Milliarden DM an Flipperautomaten ausgegeben.

Labordiagnostik gilt in Westdeutschland als technisch-medizinische Verrichtung. Für viele Menschen, auch für solche in entscheidenden Positionen, ist das etwas weniger Wichtiges, Geringwertiges, möglicherweise überhaupt keine ärztliche Aufgabe. Die mathematische Definition eines biologischen Vorganges losgelöst von Menschen, ausgeführt an seinen Körpersäften oder Gewebszellen, einer Maschine überantwortet und in einem Zahlenwert in mg dargestellt, ist etwas Abstraktes, Unpersönliches, Maschinelles. - Ist es wirklich so?

Die überwiegende Zahl von Laboruntersuchungen wird in den Praxen der Allgemeinärzte, Internisten, Kinderärzte, Urologen und Frauenärzte durchgeführt. Die Laborfachärzte haben von der Zahl der Untersuchungen nur einen Anteil unter 5\%, d. $h$. vorwiegend ganz spezielle Untersuchungen. Daraus wird deutlich, daß 95\% der Leistungen direkt in der Praxis der behandelnden Ärzte anfällt. Davon entfallen wiederum $85 \%$ auf die bekannten häufigsten Leistungen, und etwa $50 \%$ sind rationalisierbar, d.h. der analytische Teilschritt ist mit Hilfe von hochwertig technischen Geräten erbringbar.

Haematologische- und Stoffwechselwerte sind variable schnell veränderliche Werte. Wechselt z. B. der Patient den Arzt oder vom Arzt ins Krankenhaus, so wird sich nicht vermeiden lassen, daß erneut gemessen wird. Auch ist das Bedürfnis nach Laborinformation als Folge der Multimorbidität beim älteren Menschen größer als beim Jugendlichen. Das Krankenhaus mit Intensivmedizin, Reanimation und Schwerstkranken hat andere Ansprüche als die Praxis.

Während bisher die Tendenz herrschte, immer mehr Information durch mehr Zahlen zu erlangen, setzt sich langsam die Erkenntnis durch, daß dies ein Irrweg mit vielen Nachfolgekosten war. Der Rundumschlag nach der Regel - viel hilft viel - ist unergiebig. Besser scheint die gezielte Diagnostik zu sein. Wer mit offenen Augen durch die Medica geht, wird dieses bestätigt finden. Dennoch werden gewrisse Zahlen nicht zu übersehen sein. Ein gemeinschaftliches Laboratorium von 80 Ärten untersucht täglich 500 Serumproben auf 6-8 Parameter. Ein Krankenhauslaboratorium - 1 Chefarzt, 1 Assistenzarzt und ca. 15 Mitarbeiter (ohne Verwaltung) erbringt im Jahr bis zu 1,5 Millionen klinisch-chemische Leistungen. Die Kosten für einen Parameter in der Praxis liegen zwischen 2,50 DM und 3,00 DM. Das ist nur noch zu Lasten der Qualität zu unterschreiten. - Auch die Trockenchemie kann das nicht billiger machen. Der Gesetzgeber hat diese Entwicklung ausdrücklich gefördert und willentlich ausgelöst ( $\$ 368 n$, Abs. 8 der RVO). - Akzeptieren wir seinen Auftrag kritisch, so kann auch Maßstab wiederum nur die Indikation sein, ergänzt durch die Qualität und die Qualifikation des Erbringers. - Betrachten wir diesen Komplex als Grundlage für die Kosten-Nutzungsrechnung, so scheint nur diese Möglichkeit gegeben, die inflationistische Mengenausweitung in den Griff zu bekommen. Das System hat nur noch eine schwache Stelle, mit der wir alle zur Zeit Probleme haben, das ist die Automatisierung in Verbindung mit der Computerisierung. - Wir haben bisher schon wertvolle Anregungen aus der Industrie, von den Geräteherstellern bekommen. Leider manchmal erst dann, wenn die Konzeption schon fertig war und sich an der Medizin vorbei entwickelt hatte. Der Verkaufszwang tat nun sein Weiteres. Mehr gegenseitige Offenheit sollte hier Fehlentwicklungen vermeiden lassen. Auch sollte der Staat sich aus dem Dialog der Fachleute mehr als bisher heraushalten. Das hätte uns allerlei Kummer erspart.

Bleibt nur noch die Frage, ist Technik in der Medizin wirklich so inhuman und unärztlich wie ich es täglich lese, höre, sehe und wie die Medien es meiner Umgebung vermitteln? Ist die Gewebetypenbestimmung als Vorbereitung einer Organtransplantation im Laboratorium eine ärztliche Tätigkeit oder eine technische? Kann ich diese Aufgabe überhaupt erfüllen, wenn ich nicht schon viele Hunderte, von Blutgruppenbestimmungen gemacht habe und große Erfahrungswerte gespeichert habe? Ist eine einzelne Analyse sicherer als eine Analyse in einem großen Vergleichsansatz? Das letztere ist der Fall, sie ist nicht nur billiger und genauer, sie ist auch nicht mehr exklusiv, sie steht jedem zur Verfügung.

Ist es inhuman, einen Krebs mikroskopisch technisch zu diagnostizieren, wenn er noch operabel klein ist oder besser zu warten, bis ich ihn mit dem ärztlichen Finger tasten kann? Ich glaube, der Nutzen des Labors ist dann am größten, wenn es voll und ganz mit all seinen erprobten Verfahren als ärztliche Tätigkeit anerkannt wird, und wenn wir ärztlichen Maximen folgen und zukünftig folgen dürfen.

Technik ist nicht grausam, sie ist auch nicht teuer, sondern nur zeitgemäß - entscheidend ist, daß wir sie sinnvoll anwenden. Es gibt nichts humaneres als ein mit technischen Mitteln 
gerettetes oder erhaltenes gesundes Überleben. Lediglich unsere Unfähigkeit, das technische Geschehen unseren Mitmenschen richtig darzulegen, läßt die technische Medizin unmenschlich erscheinen.

Ich fasse zusammen:

Labordiagnostikistals medizinische ärztlicheAufgabe kosteneffektiv, vorausgesetzt, sie wird unter Verwendung der besten Technik, nach der Indikation und der Schutzbedürftigkeit von Patient und Arzt qualifiziert erbracht. - Wettbewerbsgedanken, Werbung und gewinngestützte Leistungsvermehrung sind folgerichtig nicht mit einer kosteneffektiven Leistungserbringung zu vereinen.

Alle von der Indikation abweichenden Maßnahmen müssen die Kosten-Nutzen-Relation zur Kostenseite hin verschieben. Medizintechnik, losgelöst von der Kontrolle und Interpretation des Arztes, wirkt auf den kranken Menschen kalt und beängstigend. Dies könnte ebenfalls durch die vorbehaltslose gleichwertige Anerkennung der Medizintechnik als ärztliche Aufgabe und die stärkere Einbeziehung des Laborarztes sowie des Mikrobiologen und Epidemiologen in die Behandlungsberatung vermieden werden.

Wenn wir seit 1957 pränatale Erkrankungen an Röteln, Lues, Masern, Varizellen, Toxoplasmose, Listeriose und Erythroblastose bis auf einige wenige tragische Fälle ausmerzen konnten, dann ist das zwar nur ein kleiner Erfolg gemessen an den Kosten, ein großer, gemessen an dem gewonnenen Leben.

In 25 Jahren mußten dafür 15-20 Millionen Schwangere untersucht werden. Bei aller aufgewandten und berechtigt eingesetzten Medizintechnik ist das eine imponierende humanitäre Leistung. Es hat Freude gemacht, daran mitarbeiten zu können, und mein Dank gilt den vielen direkten und indirekten Mitarbeitern in den ärztlichen Praxen und in den Büros und Forschungslaboratorien der Industrie.

Erinnern wir uns an dieser Stelle auch daran, daß in der Kosten-Nutzen-Betrachtung Labordiagnostik noch immer einen beachtlichen Faktor als Dienstleistung mit vielen interessanten Arbeitsplätzen darstellt.

Schrittum:

WERNER, H.: Sind obligatorische Toxoplasmose-Untersuchungen im Rahmen der Schwangerschaftsvorsorge sinnvoll? Bundesgesundheitsblatt 26, 343 (1983). .

Anschrift des Verfassers:

Dr. med. Otto Fenner

Bergstraße 14

D.2000 Hamburg 1

\section{Buchbesprechungen}

\section{Taschenbuch der Inneren Medizin}

Claus Werning und Walter Seitz, Wissenschaftliche Verlagsgesellschaft Stuttgart, 9. Auflage, ISBN 3-8047-0700-9, Preis DM 68, -

Das als Kompendium für Ärzte und Studenten konzipierte Buch erlaubt durch seine klare Gliederung, die detaillierte Inhaltsangabe und das umfangreiche Register ein schnelles Auffinden interessierender Einzelheiten und eignet sich somit gut auch als Nachschlagewerk.

Es stellt die behandelten Krankheiten knapp und komprimiert, doch flüssig lesbar dar, wobei durch die Beschränkung auf eine Auswahl relevanter Krankheitsbilder Ballast vermieden wird.

Die einzelnen Erkrankungen werden mit Pathogenese und Leitsymptomen, gegebenenfalls mit Stadieneinteilung und den sie begünsti- genden Risikofaktoren vorgestellt, die diagnostischen Verfahren in ihrer Wertigkeit und Aussagekraft angesprochen und zum Teil Kriterien zur differentialdiagnostischen Unterscheidung erwähnt.

Neben der Erläuterung von Behandlungsprinzipien und Prophylaxe stellt das Buch die einzelnen therapeutischen Maßnahmen mit Indikation, Kontraindikation, Dosierung von Medikamenten und Komplikationsmöglichkeiten dar, sowie gegebenenfalls begleitende Maßnahmen wie Anweisungen zur allgemeinen Lebensführung des Patienten. Fast durchweg sind die Angaben zu Diagnostik und Therapie auf dem neuesten Stand.

Das Buch besticht durch das harmonische Gleichgewicht zwischen theoretischen Darlegungen und praktischen Hinweisen, seinen knappen, klaren Stellungnahmen, sowie seiner Informationsfülle, deren Abrufen durch die Textgestaltung, Grafiken und instruktiven Tabellen sehr erleichtert ist. Es ist sowohl zum ersten Überblick über die Innere Medizin, wie auch als Erstinformation bei gezielten Fragestellungen sehr zu empfehlen.

D. Jüngst

\section{Ärztliches Berufsrecht - Ausbildung, Weiterbildung, Berufsausübung}

\section{Von H. Narr, Losesblattwerk in 2 Ordnern}

Das zweibändige Standardwerk von Prof. Dr. jur. H. Narr zum ärztlichen Berufsrecht wurde nunmehr durch die 5. Ergänzungslieferung hinsichtlich des Ausbildungs-, Prüfungs- und Weiterbildungsrechts einschließlich der Rechtsprechung auf den neuesten Stand gebracht. „Ärztliches Berufsrecht” gilt als anerkanntes Standardwerk zum allgemeinen ärztlichen Berufsrecht, das in keiner Handbibliothek derjenigen Juristen fehlen darf, die sich mit Fragen des allgemeinen Arztrechtes auseinanderzusetzen haben. Narr hat es erneut vorzüglich verstanden, die außerordentliche Materialfülle des ärztlichen Berufs rechts klar zu gliedern und dogmatisch einwandfrei zusammenzufassen. Die oftmals vielschichtigen und verzweigt erscheinenden Rechtsquellen - angefangen von Satzungsnormen mit landesweiter Gültigkeit, über ordentliche Gesetze bis hin zu höchstrichterlicher Rechtsprechung und Rechtsprechung des Bundesverfassungsgerichts - sind systematisch gegliedert, inhaltlich plausibel einander zugeordnet und auch im Detail zutreffend dargestellt.

Im ersten Kapitel wird die Ausbildung zum Arzt abgehandelt. Bundesärzteordnung und Approbationsordnung werden hier ebenso umfassend besprochen wie im zweiten Kapitel die Weiterbildung einschließlich der Definitionen der Gebiete, der Teilgebiete, den Weiterbildungszeiten sowie den Inhalten von Weiterbildungen. Insofern handelt es sich bei "Ärztliches Berufsrecht" gleichzeitig um ein Nachschlagewerk für den Arzt, der sich über Inhalte seines oder anderer Gebiete informieren möchte. Entsprechende Informationen gibt das Standardwerk von Narr auch über die Regeln der ärtlichen Berufsausübung, wobei unter Heranziehung einschlägiger Rechtsprechung die Grundsätze der ärztlichen Berufsausübungen dargestellt werden. Auch hier bleibt meines Erachtens keine Frage offen Der Bogen seiner Kommentierung spannt sich von den Grundsätzen ärztlicher Schweigepflicht über die Zusammenarbeit von Ärzten bis hin zu Fragen der Aufklärungspflicht, des ärztlichen Kunstfehlers, abe auch der Grundsätze über die Niederlassungsfreiheit, ärztliche Aufzeichnungen und Fragen der Ausbildung von Mitarbeitern in de Praxis. Erwähnt sei ebenfalls der Anhang, in dem sich Musterverträge zur Gründung von Gemeinschaftspraxen, zur Praxisübernahme oder auch zur Schiedsvereinbarung befinden.

Für denjenigen, der dieses Standardwerk zum ersten Mal in die Hand nimmt, wird der Wert von "Ärztliches Berufsrecht" auch durch das umfangreiche und vielschichtige Stichwortverzeichnis offenbar, das eine schnelle und zielgerichtete Orientierung ermöglicht.

Die 5. Ergänzungslieferung gibt an mehreren Stellen für Laborärzte relevante Rechtsfragen zutreffend wieder. Die offensichtliche umfassende Erfahrung und die hervorragenden rechtlichen Kenntnisse des Verfassers erlauben es ihm, schlüssig und überzeugend die Charakteristika laborärztlicher Tätigkeit darzustellen. Auch in Detailfragen kommt er zu sachgerechten und überzeugenden Ergebnissen. So führt Narr beispielsweise aus, daß ein nach einer Analyse vorliegendes Ergebnis oftmals für den diese Untersuchung veranlassenden einsendenden Arzt ohne umfassende Aussage sei. Die dort enthaltenen Informationen würden erst und nur dadurch verwendbar, daß der Arzt für Laboratoriumsmedizin hierzu einen ärztlichen Befund erstelle und damit durch die Konklusion technischer Analysenergebnisse mit 
ärztlichem Sachverstand eine ärtliche Diagnose stelle oder einen ärztlichen Therapievorschlag unterbreite.

Der Verfasser entwickelt bei der Darstellung des freiberuflichen Charakters laborärztlicher Tätigkeit überzeugend das Spannungsverhältnis zwischen dem gezielten Einsatz technischer Hilfsmittel und nichtarztlicher Mitarbeit einersoits und dem hierdurch freiwerdenden entscheidenden Merkmal der ärztlichen Befundungskapazitat des Laborarztes anderersoits. Hierdurch soi es möglich, daß sich der Laborazt auf die ollein entschoidende, eigenverantwortlich auszuubende arztlıche Tatigkeit konzentrieren könno. Es schließst sich die zutreffende und crschopfende Besprechung der hierzu einschlägigen Rechtsprechung des Bundesfinanzhofes an.

Aus laborarzllicher Sicht ist daruber hinaus von größtem Interesse die Darstellung der Unzulossigkeit einer Honorarbeteiligung von anderen Krankenhausarzten an den Vergutungen, dio ein im Krankenhaus tatiger leitender Laborarzt erhält. Die Unzulassigkeit folge insbesondere aus $\$ 18$ Musterberufsordnung, nach dem es einem Arzt nicht gestattet ist, für die Zuweisung von Patienten sich ein Entgelt oder andere Vorteile versprechen oder gewähren zu lassen oder sich selbst zu gewähren. Manche Bemühungen mit Internisten, Krankenhausverwaltungen oder Landesärztekammern wären sehr erleichtert worden, waren diese Ausführungen schon in den vorhergehenden Ergänzungslieferungen des Standardwerks von Narr enthalten gewesen.

Insgesamt ein überzeugend umfassendes und auch im Detail zutreffendes Werk. Es bleibt zu wünschen, daß in einer der zukünftigen Ergänzungslieferungen auch solche Rechtsfragen dargestellt werden, die bislang durch Parteigutachten mehr vernebelt denn geklärt erscheinen. Zu denken ist z. B. an ,Die Fiktion der Eigenerbringung“ bei der Tätigkeit von Laborgemeinschaftern. Das Standardwerk von Narr gibt auch den Nichtjuristen verläßlich Auskunft. Eine Besprechung des Standardwerks von Narr kann der dort verarbeiteten Stoffülle und dem dort durchgängig gezeigten fachlichen Niveau nur schwer gerecht werden.

H. Reineck

\section{Mitteilungen}

\section{Ringversuch: Enzymaktivitäten im Harn}

Im November 1983 führte das Institut für Toxikologie der Bayer AG in Abstimmung mit der Arbeitsgruppe "Klinische Chemie bei Laboratoriumstieren" einen Ringversuch für Enzymaktivitäten im Harn durch. Voraussetzung war die Herstellung eines für den Versand über größere Entfernungen geeigneten Kontrollmaterials in den Laboratorien des Institutes für Toxikologie der Bayer AG. Zum Versand kam humaner Urin, der Aktivitäten der Lactatdehydrogenase, $\gamma$-Glutamyltransferase, Alkalischen Phosphatase, $\beta-N$-Acetyl-D-Glucosaminidase und der Arylaminidase (sog. Alanin- bzw. Leucinaminopeptidase) enthielt, an 13 Teilnehmer.

Für mit vergleichbaren Methoden ermittelte Aktivitäten wurden Variationskoeffizienten zwischen 17\% ( $\gamma$-GT) und 32\% (LAP) erzielt.

Um die Aussagekraft zu erhöhen, soll der Ringversuch mit einer größeren Teilnehmerzahl wiederholt werden.

Interessenten für die Teilnahme an dem zunächst kostenfreien Ringversuch werden gebeten, sich bis 1. Mai 1984 an PD Dr. Dieter Maruhn oder Dr. Klaus Wehling, Bayer AG, Institut für Toxikologie, Postfach 101709, 5600 Wuppertal 1 , Tel. 0202/368263 zu wenden.

\section{INSTAND-Förderpreis}

Das Institut für Standardisierung und Dokumentation im Medizinischen Laboratorium e. V. (INSTAND) verleiht einen INSTAND-Förderpreis im Jahre 1984. Als Bewerber sind Ärzte und Naturwissenschaftler aus dem in- und Ausland zugelassen. Die einzureichenden Arbeiten sollen sich mit der Verbesserung von Analysenverfahren einschließlich ihrer Qualitätsüberwachung in der Laboratoriumsmedizin oder mit der Überwachung von meßtechnischen Anforderungen oder mit Modellen zur statistischen Auswertung von Ringversu- chen befassen. Der Preis ist mit DM 10.000, - dotiert. Die Arbeiten sollen möglichst in deutscher Sprache abgefaßt und unveröffentlicht sein. Bewerbungen sind in dreifacher Ausfertigung an Prof. Dr. med. H. Reinauer, Diabetes-Forschungsinstitut, Auf'm Hennekamp 65, 4000 Düsseldorf 1 zu richten. Einsendeschluß ist der 31. September 1984.

\section{AIDS: Wissenschaftlicher Förderpreis}

Eine bisher unbekannte Erkrankung des Immunsystems, AIDS (acquired immune deficiency syndrome oder erworbenes Immundefektsyndrom), sorgt für Beunruhigung und breite wissenschaftliche Diskussion.

Weltweit werden die Forschungsbemühungen um die Suche nach den Ursachen intensiviert.

Um die wissenschaftliche Arbeit deutscher Ärzte und Naturwissenschaftler zu würdigen, haben die Firmen MSD Sharp \& Dohme GmbH, München, und Behringwerke AG, Marburg, einen wissenschaftlichen Förderpreis ausgesetzt.

Der Preis wird mit DM 20000, - für die Jahre 1983, 1984 und 1985 festgelegt. Ausgezeichnet wird die beste deutsche Arbeit über die Pathogenese, Epidemiologie, Klinik oder Therapie des AIDS. Die Möglichkeit einer Preisteilung besteht, falls zwei gleichwertige Arbeiten eingereicht werden.

Der Preis wird durch die Deutsche Vereinigung zur Bekämpfung der Viruskrankheiten (DVV) verliehen, die auch die Jury zur Auswahl der entsprechenden Arbeit stellt.

Abgabeschluß für das Jahr 1983 ist der 30. Juni 1984.

Bewerbungen können an das Sekretariat der DVV,

Prof. Dr. Friedrich Deinhardt,

Pettenkoferstr. 9a, D-8000 München 2, gerichtet werden.

\section{Aus dem DIN \\ Deutsches Institut für Normung e.V.}

Unter dieser Rubrik soll auf neue Normentwürfe hingewiesen werden. Auf einen Abdruck des gesamten Entwurfs soll aus Platzgründen verzichtet werden, es sei denn, daß mehrheitlich eine vollständige Veröffentlichung gewünscht wird.

Für Auskünfte steht die Schriftleitung zur Verfügung.

\section{Medizinische Mikrobiologie}

Entwurf November 1983: Tuberkulosediagnostik

DIN 58943 Teil B. Empfindlichkeitsprüfungen von Tuberkelbakterien gegen Chemotherapeutica.

Es wird unter anderem ausführlich die Herstellung der chemotherapeuticahaltigen Kulturmedien, die Keimeinsaat, das Ablesen und die Beurteilung behandelt.

Einspruchsfrist: 29. Februar 1984.

Entwurf Dezember 1983: Medizinisch-mikrobiologische Laboratorien

Beiblatt 1 zu DIN 58956 Teil 1.

Zuordnung von Mikroorganismen zu Risiko-Gruppen I bis IV. Hier werden wichtige humanpathogene Viren, Bakterien, Pilze und Parasiten den in DIN 58956 Teil 1 definierten vier Risikogruppen zugeordnet. Die vier Gruppen wurden nach Einschätzung der bei Arbeiten mït diesen potentiellen Krankheitserregern möglicherweise auftretenden Gefährdungen definiert.

Stellungnahmen bis 31. März 1984. 


\section{Deutsche Gesellschaft für Laboratoriumsmedizin - Arbeitskreis MTA}

Der Arbeitskreis MTA der Deutschen Gesellschaft für Laboratoriumsmedizin ist inzwischen zu einem stehenden Begriff geworden. Es war sicher ein richtungweisender Schritt, daß aufgrund $\$ 4,3$ der neuen Satzung der Gesellschaft MTA und MTLA mit Erlaubnis zum Führen ihrer Berufsbezeichnung außerordentliche Mitglieder werden können. Sie können zu den Sitzungen des Gesamtvorstandes hinzugezogen werden. Bei den Mitgliederversammlungen haben sie Teilnahme- und Beratungsrecht, und sie sollen von den Organen der Gesellschaft in eigenen Angelegenheiten gehört werden.

Aus der klaren Erkenntnis heraus, daß die MTA die wichtigsten Mitarbeiter der Laborärzte sind, aus der Tatsache, daß Laborärzte Leiter und Dozenten sowohl an MTA-Ausbildungsstätten wie bei Weiterbildungsmaßnahmen sind, hat es schon seit Jahren Kontakte und gemeinsame Besprechungen über Fragen gegeben, die beide Berufsgruppen berührten. Es gelang nunmehr in Gestalt des Arbeitskreises MTA eine ständige Einrichtung zu schaffen, in der in offener Diskussion alle anliegenden Fragen besprochen und Richtlinien sowie Empfehlungen erarbeitet werden können, die anschließend den beiden Vorständen zur Entscheidung vorgelegt werden. Dabei werden möglicherweise kontroverse Themen nicht ausgelassen. Es zeigte sich aber schon bei den bisherigen Sitzungen, daß die Interessen im allgemeinen in die gleiche Richtung weisen. Die Sitzungen des Arbeitskreises sind öffentlich und die Teilnahme damit für jedes Mitglied möglich. Unter der Leitung von Prof. Dr. Rausch-Stroomann gehören von Anfang folgende Herren dem Arbeitskreis an: Prof. Linzenmeier, Prof. Ringelmann, Prof. Paar, Prof. Primavesi. Dieser Kreis ist im Herbst 1983 um folgende Mitglieder enweitert worden: Dr. Bergner, Dr. vom Dahl, Prof. Diefenthal, Dr. Gütgemann, Prof. Paschen, Prof. Thomitzek und Prof. Walter.

Von seiten des dvta waren bisher Frau Schmidt, Frau Schramm, Frau Brett, Herr Jochmann und Frau Zimmermann vertreten. An der deutlich steigenden Teilnehmerzahl bei den Sitzungen ist ein zunehmendes Interesse zu erkennen.

Bisher haben 4 Sitzungen stattgefunden, davon 2 in Bad Nauheim (1982 und 1983) sowie je eine in Wien und in Düsseldorf.

Einstiegsthema bei der konstituierenden Sitzung am 30. 10. 1982 war die Frage der Finanzierung der MTA-Lehranstalten in der Bundesrepublik, die zu dem damaligen Zeitpunkt auf das äußerste gefährdet war. Schon war es zur Schließung einzelner Schulen gekommen.

Der Arbeitskreis arbeitete eine Resolution aus, die noch am gleichen Tag dem Gesamtvorstand der Deutschen Gesellschaft für Laboratoriumsmedizin vorgelegt und verabschiedet, sowie unter dem 25.11. 1982 dem BMJFG, den Ministerien für Arbeit der Bundesländer und der Deutschen Krankenhausgesellschaft zugestellt wurde. Inzwischen hat sich die Lage auf diesem Sektor deutlich entspannt, bedarf aber weiterhin aufmerksamer Beobachtung. Der Zeitpunkt der Gründung des Arbeitskreises war aber auch aus anderen Gründen wie vorprogrammiert und dringlich, denn soeben hatte der dvta seinen Entwurf zur Novellierung des MTA-Gesetzes vom 8. 9. 1971 vorgelegt, der schon bei einigen anderen Berufsverbänden Aufsehen erregt hatte und der sich vorerst wie ein roter Faden durch alle Sitzungen des Arbeitskreises MTA gezogen hat und weiterhin ziehen wird.

Über die Verlängerung der Ausbildungszeit für MTA auf 3 Jahre herrscht im Arbeitskreis Übereinstimmung.

Die eindeutigen Vorteile liegen in einer besseren Fundierung des Basiswissens, sowie in der EG-Konformität. Das theoretische Wissen sollte nicht um, neuen Stoff erweitert, wohl aber vertieft vermittelt werden. Die praktische Ausbildung jedoch sollte deutlich vermehrt werden. Im Hinblick auf die sehr unterschiedliche Qualität der MTA-Schulen in der BRD kann man sicher mit der Zustimmung aller Laborärzte, die mit MTA arbeiten, rechnen.

Abschließend und auch bei der Sitzung in Düsseldorf am 18. 11.1982 wurde über die weiteren Inhalte des Novellierungsentwurfs diskutiert. Auch der Vorsitzende Prof. Reinauer nahm an den beiden Sitzungen teil. Wenn man davon ausgeht, daß das MTA-Gesetz, wie schon bisher die Tätigkeit verschiedener Berufsgruppen regelt und weitgehend mitbestimmt, wünscht man natürlich klare Definitionen und unmißverständliche Formulierungen bereits im Gesetzestext und nicht in Kommentaren oder Fußnoten.

Solche „heißen Begriffe“ sind z. B. die „selbständige Tätigkeit" oder „vorbehaltene Tätigkeiten”.

Sicher können sich gerade aus der Arbeit des Arbeitskreises in Verbindung mit dem Vorstand wertvolle Anregungen ergeben, die sowohl für die zukünftige Arbeit der Laborärzte, wie der MTA, im Sinne einer Abgrenzung gegenüber anderen Berufsgruppen brauchbare Hilfe darstellen können.

Weiteres Thema in Düsseldorf war die Anerkennung von MTA-Examina außerhalb des Geltungsbereichs des MTAGesetzes.

Diese Frage sollte nach einem Schreiben von Prof. Reinauer beim Treffen der Gesundheitsdezernenten der Länder besprochen werden.

Schließlich wurde über die Weiterbildung der MTA zum Fachassistenten referiert, wie sie unter Leitung des Deutschen Institutes zur Weiterbildung MTA, Sitz Berlin seit ca. 12 Jahren betrieben wird. Z.Zt. werden die Ausführungsbestimmungen für das Fach Klinische Chemie zum Berliner Gesetz über die Weiterbildung in den Medizinalberufen (WMfG) vom 9. 2.1979 vorgelegt. Dieses soll den Absolventen aller Fachassistentenkurse die staatliche Anerkennung ihrer enworbenen Diplome bringen. Inzwischen ist die Deutsche Gesellschaft für Laboratoriumsmedizin auch Mitglied des Beirates des DIW-MTA geworden.

Die Sitzung in Wien anläßlich der Kongresse der Gesellschaften für Laboratoriumsmedizin aus Deutschland und Österreich und der österreichischen Gesellschaft für Klinische Chemie fand am 22. 4. 1983 statt. Sie diente in erster Linie einem Erfahrungsaustausch zwischen den beiden Ländern, der sich vorwiegend auf die. MTA bezog, aber für die Laborärzte 
bestimmt nicht uninteressant war. Folgende Themen wurden diskutiert :

1. Zusammenarbeit Laborarzt/MTA.

2. Probleme der MTA-Schulen,

3. Weiterbildung der MTA.

In dem Labor ciner ltd. MTA von Osterreich waren 2. B. 7 Ärzte latig ( 4 ausgebildete und 3 in der Ausbildung), ferner 16 MTA. An 4 Wochentagen haben die MTA Nachtdienst, an 3 Tagen die Arzte. Die MTA arbeitet in ärztlichem Auftrag, selbständig und nicht unter Aufsicht. Ein größeres Labor darf ausschließlich von einem Facharzt für Laboratoriumsmedizin geleitet werden.

In einem kleineren Krankenhaus kann auch ein Internist oder der Pathologe Leiter des Labors sein.

Ab 7-8 MTA gibt es eine leitende MTA mit definierten Aufgaben. Für die MTA-Ausbildung wird in Österreich das Abitur benötigt. Es gibt keine Privatschulen, Schulgeld wird nicht erhoben. Schulleiter muß ein Arzt sein.

Erwähnenswert war noch die eigene Aktivität der MTA in Osterreich bei eigenen Kongressen oder mit anderen Berufsgruppen oder parallel zu anderen auch internationalen Kongressen.

Die letzte Sitzung des Arbeitskreises MTA fand am 30. 10. 1983 anläßlich der Herbsttagung der Gesellschaft in Bad Nauheim statt. Diesmal beschäftigte sich der Arbeitskreis mit Fragen der Labororganisation.

In Zeiten der Kostenrestriktion im Gesundheitswesen und anhaltender Verteilungskämpfe ist es wichtig für bestimmte Positionen (Stabsstellen) eine Art Stellenbeschreibung zu erarbeiten, die nicht etwa dazu dienen soll, Laborarzt und MTA auseinanderzudividieren - das ist mit Sicherheit nicht das Ziel unseres Arbeitskreises.

Erst die Auflistung der Aufgaben z.B. eines Laborleiters (Laborfacharzt) sowie der leitenden MTA macht vor allem Dritten gegenüber klar, wie vielseitig diese Aufgaben sind und wie sehr es dabei einer Arbeitsteilung bedarf. Diese ist natürlich von Labor zu Labor recht unterschiedlich, so daß diverse Aufgaben entweder vom Laborchef oder von der leitenden MTA übernommen werden können. Es war sicher nicht unser Ziel, hier feste Grenzen zu setzen oder alles festzuschreiben. Im Endeffekt ergibt sich, daß eine vertrauensvolle Zusammenarbeit für das Wohl und Wehe des Labors sehr wichtig ist.

Die sehr zahlreichen und heterogen zusammengesetzten Teilnehmer führten eine engagierte Grundsatzdebatte über das Vorhaben des Arbeitskreises und beschlossen, dem Vorschlag zu folgen. Anhand einer Tischvorlage, die noch einmal an alle Teilnehmer verschickt wurde, wurden die einzelnen Punkte durchgegangen und die endgültige Fassung sowie die Verabschiedung der Tätigkeitsmerkmale auf die nächste Sitzung verschoben. Dabei sollen auch noch andere Berufsgruppen, die im Labor tätig sind, mit berücksichtigt werden.

Als Präambel des Aufgabenkataloges wurde formuliert:

Die verantwortliche Laborleitung liegt in den Händen des Laborarztes. Die Itd. MTA kann unter seiner Weisung und Aufsicht, Tätigkeiten, die eigentlich der ärztlichen Laborleitung zustehen, auf dem Delegationsweg übernehmen.

Der leitende Laborarzt hat das Recht, sich jederzeit bestimmte Tätigkeiten aus dem Katalog der Itd. MTA vorzubehalten, je nachdem wie er es für sein Labor für notwendig erachtet.

Abschließend wurde diskutiert, ob der dvta auf Kongressen der Fachgesellschaft mit eigenem Programm oder eigenen Referenten teilnehmen sollte.
Nach Zustimmung des Vorstandes der Gesellschaft soll dieses im Bundesvorstand des dvta beraten werden.

Wenn die Aktivitäten des Arbeitskreises MTA insgesamt betrachtet werden, so läßt sich sagen, daß ein erfreulicher Anfang zur Verfolgung gemeinsamer Ziele zweier Berufsverbände gemacht wurde.

Prof. Dr. J.-G. Rausch.Stroomann

Abt. für Laboratoriumsmedizin

Kreiskrankenhaus Lemgo

D.4920 Lemgo

\section{Tagungen}

St. Louis, Missouri, USA: Während des 84th Annual Meeting of the American Society for Microbiology" vom 4. bis 9. März 1984 findet am 6. März 1984 ein Seminar mit dem Titel .,Recent Developments for Detecting Endotoxins in Body Fluids" statt.

Auskunft:Prof. Dr. Bernhard Urbaschek, D 6, 5, 6800 Mannheim. Tel. $0621 / 102097$

München: Vom 7. bis 9. März 1984 findet in München das 3rd International Symposium on Inborn Errors of Metabolism in Humans statt.

Auskunft: I. Berner, Kongreßsekretariat, Med. Poliklinik, Pettenkoferstraße 8a, 8000 München 2, Tel. 089/51 60-3500.

Bad Hofgastein : Die Dreiländertagung der Deutschen Gesellschaft für Zytologie, Schweizerischen Gesellschaft für Zytologie und Österreichischen Gesellschaft für angewandte Zytologie findet vom 23. bis 29. März 1984 statt.

Themen: Gynäkologische Zytodiagnostik. Zytodiagnostik der Mamma. Zytologie der Effusionen. Zytologie der Prostata. Zytologie der Harnwege.

Auskunft: OA Dr. K. Czerwenka, Institut für pathologische Anatomie, Spitalgasse 4, A-1090 Wien, Tel. 0222/4800-3691.

Innsbruck: Am 30. und 31. März 1984 veranstaltet die Österreichische Gesellschaft für Klinische Chemie ein Seminar „Säure-, Basenund Elektrolythaushalt".

Auskunft: Prim. Dr. W. Hohenwallner, Nöbauerstraße 25, A-4060 Leonding, Tel. 0732/275181.

St. Louis (USA) : Vom 1. bis 6. April 1984 findet das Annual Meeting der Federation of American Societies for Experimental Biology statt.

Themen: Research in Physiology / Pharmacology / Biochemistry / Nutrition / Immunology.

Auskunft: Office of Scientific Meetings, 9650 Rockville Pike, Bethesda, MD 20814 USA.

Zur gleichen Zeit tagt dort die American Association of Immunologists (siehe Lab.med, 7 (1983), A+B 171).

Düsseldorf: Die 8. Düsseldorfer Hygienetage finden am 5. und 6. April statt.

Themen: Aktuelle Infektionen und ihre Prophylaxe. Aktuelle Probleme der Lebensmittelhygiene.

Auskunft: Hans Pietsch, Henkel KGaA, Stabsstelle Öffentlichkeitsarbeit, Postfach 1100, 4000 Düsseldorf 1, Tel. 0211/7973735.

Bad Harzburg: Vom 10. bis 13. April 1984 findet die 11. Tagung der Deutschen Gesellschaft für Parasitologie e.V. statt.

Themen: Epidemiologie der Parasitosen des Menschen und der Tiere. Parasitenstoffwechsel - In vitro-Kultur - Arzneimittelresistenz.

Auskunft: Dr. D. Düwel, Deutsche Ges. f. Parasitologie e.V., Postfach 800320,6000 Frankfurt/M. 80. 
Mosbach: Das 35. Mosbacher Kolloquium findet vom 12. bis 14. April 1984 statt.

Thema: The Impact of Gene Transfer Techniques in Eucaryotic Cell Biology.

Auskunft: Prof. Dr. J. Schell, Max-Planck-Institut für Züchtungsforschung, Egelspfad, 5000 Köln 30 oder Prof. Dr. P. Starlinger, Institut für Genetik, Weyertal 121, 5000 Köln 41.

München: Vom 16. bis 18. April 1984 findet der 3rd International Workshop on Trace Element statt.

Thema: Analytical Chemistry in Medicine and Biology.

Auskunft: Hannelore Teichmann, Rindermarkt 5, 8000 München 2 , Tel. 089/2391216

Lugano: 26. bis 28. April 1984 - 43. Jahresversammlung und Kongreß der Schweizerischen Gesellschaft für Mikrobiologie.

Themen: Mikrobiologie und Umwelt. Importierte Krankheiten. Wasser- und Abwasserhygiene. Lebensmittelmikrobiologie (Verunreinigung der Nahrungsmittel).

Auskunft: Dr. J. D. Piguet, Institut d'hygiène, CH-1211 Genf 4.

Boston: Der III. Internationale Kongreß für Andrologie wird vom 28. April bis 2. Mai 1985 von der International Society of Andrology und der American Society of Andrology veranstaltet.

Auskunft: Robert A. Newton, M.D., Program Chairman, c/o Third International Congress of Andrology, 2000 Washington Street, Newton, MA, USA 02162.

Basel: Am 5. Mai 1984 findet die Jahresversammlung der Schweizerischen Gesellschaft für Klinische Zytologie statt.

Auskunft: PD Dr. med. P. Dalquen, Institut für Pathologie der Universität Basel, Schönbeinstraße 40, CH-4003 Basel, Tel. 252525.

Wiesbaden: 9. bis 12. Mai 1984 - 16. Tagung der Deutschen Gesellschaft für Allergie- und Immunitätsforschung.

Themen: Genetik und Immunantwort. Überempfindlichkeit auf chemische Umweltstoffe und Pharmaka. Immunmodulation - therapeutische Aspekte.

Auskunft: Prof. Dr. med. E. Fuchs, FB Allergologie, Deutsche Klinik für Diagnostik, Aukammallee 33, 6200 Wiesbaden.

Mailand: 15. bis 17.' Mai 1984: RIA '84, International Symposium on "Cost and Benefit of Radioimmunoassay". Advanced Course on (17. 5.): Ferritins and Isoferritins as Biochemical Markers: Biological and Clinical Aspects.

Auskunft: Fondazione Giovanni Lorenzini, Via Monte Napoleone, 23, I-20121 Milano. Tel. 02/783868 oder 702267 .

Gent: 15. bis 18. Mai 1984 - Fifth International Symposium on Mass Sectrometry in Life Sciences.

Auskunft: Prof. Dr. A. de Leenheer, Laboratoria voor Med. Biochemie en voor Klinische Analyse, Rijksuniversiteit Gent, Harelbeekestraat 72, B-9000 Gent.

Buxton, Derbyshire, England: 21. bis 23. Mai 1984 - ACB (Association of Clinical Biochemists) National Meeting 1984.

Auskunft: Dr. C. Toothill, Dept. of Chemical Pathology, University of Leeds, Leeds LS2 9JT, England.

Moskau: Vom 25. bis 30. Juni 1984 ist das 16 th Meeting of the European Biochemical Societies - FEBS - in Moskau.

Auskunft: 16th FEBS Meeting Secretariat, Shemyakin Institute of Bioorganic Chemistry, USSR Academy of Sciences, UI. Vavilova 32. GSP-1, Moscow V-334, 117988 USSR.

Kongreßreise durch Deutsches Reisebüro $\mathrm{GmbH}$, Eschenheimer Landstraße 25-27, 6000 Frankfurt/Main, Tel. 0611/1566-386.

Berlin: Das ,.Fourth International Symposium on Rapid Methods and Automation in Microbiology and Immunology" findet vom 7. bis 10. Juni 1984 in Berlin statt. Die unter Leitung von Prof. Dr. Habermehl stehende Veranstaltung wird unterstützt von der Deutschen Geselischaft für Hygiene und Mikrobiologie (DGHM) und der International Union of the Microbiological Societies (IUMS). Themen: Time realities in the evaluation of vaccines for safety and efficacy; hybridisation technology in microbiology: hybridisation technology in virology; rapid diagnosis by lgM-detection; application of monoclonal antibodies; problems of hybridoma technology; data processing in microbiology and serology; fluoroimmunoassays bioluminescence; physical detection of microorganisms; chromatography; antigen detection; isolation of microorganisms; identification of microorganisms; antimicrobial susceptibility tests; assays of antimicrobial agents in fluids and tissues; food microbiology; en vironmental microbiology.

Auskunft: H. Zeichhardt Institut für klinische und experimentelle Virologie der freien Universität Berlin, Hindenburgdamm 27 1000 Berlin 45, Telefon (030) 7983696/7.

\section{Terminkalender}

\section{März 1984}

4. - 9.3. St. Louis (USA): 84th Annual Meeting of the American Society for Microbiology (BDL 1984, 18)

7.- 9.3. München: 3rd International Symposium on Inborn Errors of Metabolism in Humans (BDL 1984, 18)

21.-23.3. Erfurt: Pathologische und klinische Biochemie' 84 (BDL 1984, 10)

23.-29.3. Bad Hofgastein: Dreiländertagung der Deutschen Schweizerischen und Österreichischen Gesellschaft für Zytologie (BDL 1984, 18)

30.-31.3. Innsbruck: Säure-, Basen- und Elektrolythaushalt (BDL 1984, 18)

\section{April 1984}

1.- 7.4. Obergurgl: 4. Wiss. Fortbildungstagung für Laboratoriumsmedizin (BDL 1984, 10)

1.- 6.4. St. Louis (USA): Annual Meeting FASEB (BDL 1984,

5.- 6.4. Düsseldorf: 8. Düsseldorfer Hygienetage (BDL 1984, 18)

10.-13.4. München: Analytica '84 (BDL 1984, 10)

10.-13.4. Bad Harzburg: 11. Tagung d. Deutschen Ges. f. Parasitologie (BDL 1984, 18)

12.-14.4. Mosbach: 35. Mosbacher Kolloquium (BDL 1984, 19)

16.-18.4. München: 3rd International Workshop on Trace Element (BDL 1984, 19)

26. -28.4. Lugano: 43. Jahresversammlung u. Kongreß d. Schweiz. Ges. f. Mikrobiologie (BDL 1984, 19)

29.4.-2.5. Graz: International Symposium on Prolactinomas (BDL 1984, 10)

29.4.-5.5. Rio de Janeiro: XII. Intern. Kongreß "Clinical Chemistry" (BDL 1984, 10)

30.4.-2.5. Brüssel: XXXII Annual Colloquium Protides of the Biological Fluids (BDL 1984, 10)

\section{Mai 1984}

4.- 6.5. Bremen: 18. Tagung der Deutschsprachigen Mykologischen Gesellschaft (BDL 1984, 10)

Basel: Jahrevers. Schweiz. Ges. f. Klinische Żytologie (BDL 1984, 19)

9.-12.5. Wiesbaden: 16.Tagung d. Dtsch. Ges. f. Allergie- u. Immunitätsforschung (BDL 1984, 19)

15. - 17.5. Mailand: RIA '84 (BDL 1984, 19)

15.-18.5. Gent: 5 . Intern. Symposium on Mass Spectrometry in Life Sciences (BDL 1984, 19)

21.-23.5. Buxton: ACB National Meeting 1984 (BDL 1984, 19) 22. - 24.5. Feldkirch: 19. Jahrestagung der Österreichischen Gesellschaft für Hygiene, Mikrobiologie und Präventivmedizin (BDL 1984, 10)

23.-25.5. Marseille: Fed. of European Microbiological Societies (Lab.med. 1983, A+B 171)

31.5.-2.6. Titisee: Fortschritte in der Forschung der Glucuronsäure-Konjugation (BDL 1984, 10)

\section{Eingegangene Bücher}

W. M. Nentwig: JURAMED - Recht des niedergelassenen Arztes. Ergänzbares Loseblattwerk im Sammelordner. 2. Auflage 1980 mit 4. Nachtragslieferung März 1983. Verlag Kirchheim \& Co GmbH, Mainz, DM 98, -

H. P. Seelig: Antikörper gegen Zellkernantigene. Labormedizin und Klinik, Heft 1. Hrsg.: H. P. Seelig. H. Liehr, R. Seelig. 151 S. Gustav Fischer Verlag, Stuttgart, New York 1983, DM 48, - 


\section{Gesundheitliche Richtlinien, Empfehlungen und Ratschläge des Bundesgesundheitsamtes}

Fortsetzung aus Heft 1/1984. BOL 12

Nr. Titel

25. Merkblatt Nr. 42 , Keratoconjunctivitis epidemica - Erkennung und Verhütung".

Ausgabe Marz 1975

26. Merkblatt Nr. 51 .Kopflausbefall (Pediculosis capitis) - Vorhütung und Bekämplung". Ausgabe 1983

27. Merkblatt ..Einleitung von Kranken hausabwasser in Kanalisation oder Gewässer".

Stand: 1978

28. Richtlinie für die Erkennung, Verhütung und Bekämpfung von Kran. kenhausinfektionen.

Stand: 1983

29. Empfehlungen des Bundesgesundheitsamtes zur gesundheitlichen Beurteilung von Kunststoffen im Rahmen des Lebensmittel- und Bedarfsgegenständegesetzes. Stand: 1983

30. Untersuchungen von Kunststoffen, soweit sie als Bedarfsgegenstände im Sinne des Lebensmittel- und Bedarfsgegenständegesetz verwendet werden.

Stand: 1982

31. Gesundheitliche Beurteilung von Kunststoffen und anderen nichtmetallischen Werkstoffen im Rahmen des Lebensmittel- und Bedarfsgegenständegesetzes für den Trinkwasserbereich. Stand: 1979

32. Amtliche Sammlung von Untersuchungsverfahren nach § 35 LMBG. Verfahren zur Probenahme und Untersuchung von Lebensmitteln, Tabakerzeugnissen, kosmetischen Mitteln und Bedarfsgegenständen. Stand: 1983

33. Probenvorbereitungsverfahren für die Bestimmung von Schwermetallgehalten in und auf Lebensmitteln. Stand: 1979

34. Richtwerte '79 für Blei, Cadmium und Quecksilber in und auf Lebensmitteln.

Stand: 1979

35. Richtlinien zum Schutz vor Gefahren durch in vitro neukombinierte Nukleinsäuren.

3., überarbeitete Fassung. Stand: 1980

36. Probenahmeverfahren für die Kontrolle der Rückstände von Schädlingsbekämpfungsmitteln auf und in Obst und Gemüse. Stand: 1980

\author{
Fundstelle \\ Bundesgosundheitsblatt \\ 19 (1976) $11-12^{\circ}$
}

Bundesgesundheitsblatt 26 (1983) 185-188.

Bundesgesundheitsblatt 21 (1978) $34^{\circ}$.

Gustav Fischer Verlag,

Stuttgart.

Bundesgesundheitsblatt 26 (1983) $53^{\circ *}$

Bundesgesundheitsblatt 25 (1982) $35^{* *}$.

Bundesgesundheitsblatt 22 (1979) $264^{* *}$.

Beuth Verlag, Berlin Bd. I: Lebensmittel

Bd.II: Bedarfsgegenstände

Bd. III: Kosmetik

Bd. IV: Tabakerzeugnisse

Bundesgesundheitsblatt 22 (1979) 277-279.

Bundesgesundheitsblatt 22 (1979) 282-283.

Bundesanzeiger Nr. 129 vom 17.7.1980.

Bundesgesundheitsblatt 23 (1980) 397.

\section{Nr. Titel}

37. Vorläufige Empfehlungen für den Umgang mit pathogenen Mikroorganismen und für die Klassifikation von Mikroorganismen und Krankheitserregern nach den im Umgang mit ihnen auftretenden Gefahren.

38. Empfehlungen des Bundesgesundheitsamtes zur Verzehrseinschrän. kung wegen Schwermetallgehaltes in Speisepilzen.

Stand: 1978

39. Merkblatt Nr.18 „Pocken - Verhütung und Bekämpfung". Ausgabe Januar 1976

40. Merkblatt Nr. 27 ,Infektionskrankheiten und Schutzimpfungen im internationalen Reiseverkehr". Ausgabe 1981

41. Liste der vom Bundesgesundheitsamt geprüften und anerkannten Mittel zur Mückenabwehr (Repellents).

Stand: 1983 (13. Ausgabe)

42. Merkblatt Nr.15 „Scharlach Verhütung und Bekämpfung". Ausgabe März 1973

43. Zeitabstände zwischen Schutzimpfungen: Stand: 1983

44. Empfehlung des Bundesgesundheitsamtes zur Anwendung von Solarien.

Stand: 1979

45. Richtlinien 1979 für die Serodiagnose der Syphilis.

46. Merkblatt Nr. 3 „Tollwut - Verhütung und Bekämpfung". Ausgabe April 1978

47. Merkblatt Nr. 20 ,Toxoplasmose Erkennung und Verhütung". Ausgabe Juli 1980

48. Empfehlung des Bundesgesundheitsamtes zum Grenzwert für Nitrat nach der Trinkwasserverordnung.

Stand: 1979

49. Merkblatt Nr. 21 „Virushepatitis Verhütung und Bekämpfung". Ausgabe September 1980

50. Merkblatt Nr. 26 ,Richtlinien für sonstigen Gemeinschaftseinrichtungen nach dem 6. Abschnitt des Bundes-Seuchengesetzes". Ausgabe November 1980

51. Richtlinien für die Gewinning keimfreier zellulartherapeutischer Präparate und für die Gesundheitskontrolle der Spendertiere. Stand: 1978

\section{Fundstelle}

Bundesgesundheitsblatt 24 (1981) 347-358.

21 (1978) 204.

$-\bullet$

Bundesgesundheitsblatt 24 (1981) 261-265, $335^{\circ}$

Bundesgesundheitsblatt 26 (1983) 184

Bundesgesundheitsblatt 16 (1973) $120-122^{\circ}$

Bundesgesundheitsblatt 26 (1983) 128.

Bundesgesundheitsblatt 22 (1979) 216.

Bundesgesundheitsblatt 22 (1979) 398-400.

Bundesgesundheitsblatt 21 (1978) 289-292*.

Bundesgesundheitsblatt 24 (1981) 93-96*.

Bundesgesundheitsblatt 22 (1979) 102

Bundesgesundheitsblatt 24 (1981) 12-18.

Bundesgesundheitsblatt 24 (1981) 145-147.
Bundesgesundheitsblatt 23 (1980) 153-154.

\footnotetext{
- Deutscher Árte-Verlag GmbH, Dieselstr. 2. Postfach 400440, D-5000 Köin 40.

. Mitteilungen gesammelt in . Kunststoffe in Lebensmitteln". Herausgeber: R. Franck, Carl Heymanns Verlag, Köln.
} 